\title{
Perspective
}

PERSPECTIVE Actualité en histoire de l'art

2| 2009

L'Espagne

\section{Art contemporain : le grand pari institutionnel}

Contemporay art: the great institutional gamble

José Guirao, Bartomeu Marí, Vicente Todolí et Estrella de Diego

\section{(2) OpenEdition}

Journals

Édition électronique

URL : http://journals.openedition.org/perspective/1376

DOI : 10.4000/perspective. 1376

ISSN : 2269-7721

Éditeur

Institut national d'histoire de l'art

Édition imprimée

Date de publication : 30 juin 2009

Pagination : 247-254

ISSN : 1777-7852

\section{Référence électronique}

José Guirao, Bartomeu Marí, Vicente Todolí et Estrella de Diego, « Art contemporain : le grand pari institutionnel », Perspective [En ligne], 2 | 2009, mis en ligne le 24 juin 2013, consulté le 01 octobre 2020. URL : http://journals.openedition.org/perspective/1376 ; DOI : https://doi.org/10.4000/ perspective. 1376 


\title{
Art contemporain : le grand pari institutionnel
}

\author{
Points de vue de José Guirao, Bartomeu Marí et Vicente Todolí, avec Estrella de Diego
}

Il suffit de se rappeler qu'en 1964, quand Juana Mordó ouvrait sa galerie à Madrid, elle était la seule à présenter l'art d'avant-garde du moment, I'Informalismo ou Art informel - alors qu'à la même époque les galeries des principales villes européennes et celles de New York avaient une activité soutenue -, pour prendre la mesure du triste panorama qui était celui de I'Espagne à la fin des années 1960 et au début des années 1970. Tout ce qu'a vécu ce pays depuis lors n'en est que plus remarquable, qu'il s'agisse des timides initiatives privées des années 1970 pour soutenir l'art contemporain ou de la politique instaurée dans les années 1980 pour ouvrir le pays à l'art vivant, tant par des actions gouvernementales que par l'intermédiaire des fondations qui ont joué un rôle essentiel dans la "modernisation " de la scène artistique actuelle. Ainsi, la Nouvelle Figuration, qui prend corps dans le Madrid des années 1980, devint le courant à exporter, offrant au pays une projection internationale avec une image différente de celle antérieure au franquisme. Fut ainsi créée la foire internationale d'art contemporain, ARCO, dont la première édition en 1981 permit aux professionnels espagnols d'entrer en contact avec leurs collègues étrangers. Le mouvement soutint également des jeunes prometteurs en les aidant à participer à des manifestations comme Europalia 85 à Bruxelles, ou en organisant des expositions comme El siglo de Picasso, qui s'est tenue en 1987-1988 au Centro de Arte Reina Sofía, Madrid et au Musée d'art moderne de la ville de Paris. Les fondations privées Juan March de Madrid et celle de La Caixa, ainsi que le Centro Nacional de Exposiciones del Ministerio de Cultura, n'ont pas économisé leurs efforts, dès le début des années 1980, pour présenter à Madrid les premières avant-gardes et l'art des années 1960, absents de la scène espagnole, pour des raisons évidentes, durant la dictature.

La situation de l'Espagne se normalisait peu à peu, si bien qu'à l'approche de l'année 1992 - une année clé car elle réunit plusieurs événements de dimension internationale -, tout un mécanisme se mit en marche pour donner le dernier coup de pouce au processus de changement déjà enclenché. De manière plus générale, dans la continuité du cinq centième anniversaire de la découverte de l'Amérique, de l'Exposition universelle de Séville et des Olympiades de Barcelone, les années 1990 s'avèrent fondamentales pour la modernisation définitive du pays dans le domaine artistique. Au cours de cette décennie, une grande partie des principaux musées publics d'art contemporain fut inaugurée, dont certains avaient commencé à se mettre en place à la fin
Estrella de Diego est professeur d'art contemporain à I'Universidad Complutense, Madrid et professeur invité à la New York University. Commissaire de la $22^{\mathrm{e}}$ Biennale de Sao Paulo et de la 49e Biennale de Venise, elle a organisé l'exposition Warhol sobre Warhol (Madrid, La Casa Encendida, 2007).

Actuellement directeur de La Casa Encendida, José Guirao a dirigé le Museo Nacional Centro de Arte Reina Sofía, Madrid (1994-2000), les Bellas Artes y Archivos, Ministerio de Cultura (1993-1994) et les Bienes Culturales, Junta de Andalucía (1988-1993).

Bartomeu Marí est conservateur en chef du MACBA, Barcelone depuis 2008. Il a été conservateur en chef de l'IVAM, Valence, directeur du centre d'art contemporain Witte de With, Rotterdam, et a travaillé à la Fondation pour l'architecture, Bruxelles.

Directeur de la Tate Modern, Londres depuis 2003, Vicente Todolí a fondé et dirigé le Serralves Museum of Contemporary Art, Porto (1996-2003) et a été conservateur en chef (1986-1988) puis directeur artistique (1988-1996) de I'Instituto Valenciano de Arte Moderno, Valence. 
des années 1980 : I'IVAM (Institut Valencià d'Art Modern)et le CAAM (Centro Atlántico de Arte Moderno) en 1989; le Centro Andaluz de Arte Contemporáneo en 1990 ; le Museo Nacional Centro de Arte Reina Sofía ouvert en 1991-1992; le CGAC (Centro Galego de Arte Contemporánea), le MACBA (Museu d'Art Contemporani de Barcelona) et le MEIAC (Museo Extremeño e Iberoamericano de Arte Contemporáneo) inaugurés en 1995. La structure politique particulière du pays, un État constitué de communautés autonomes, explique la dispersion des initiatives, chacun des gouvernements désirant doter ses villes d'un projet spécifique d'art contemporain capable de rivaliser avec les institutions étatiques.

C'est dans cet esprit de changement que l'Espagne est entrée dans le XXle siècle, moment où l'art vivant a pris un véritable essor. La majorité des efforts de modernisation se sont concentrés sur ce domaine au cours des dix dernières années, au détriment, il faut bien l'avouer, de l'art "moderne », c'est-à-dire les avant-gardes historiques ou même celles du XIX siècle. Le début du XXI siècle a vu culminer, il est vrai, de grands projets qui ne concernaient pas exclusivement l'art actuel : le développement du musée Reina Sofía, l'ouverture du Museo Picasso à Málaga (MPM) ou même l'agrandissement notoire du Museo Nacional del Prado. Une part de ces projets a suppléé ou devrait suppléer à certaines carences concernant le XIX siècle et l'art "moderne ", même si un gros travail reste nécessaire pour beaucoup de musées dont les collections d'art espagnol datent de la première moitié $d u$ xxe siècle. En tout état de cause, et toujours dans le cadre de ce pari pour l'art vivant, de nouveaux musées d'art contemporain ont rejoint ceux déjà existants. Aux côtés de centres qui possèdent des collections plus orientées sur le XXe siècle en général, il faut mentionner des propositions comme celle du MUSAC (Museo de Arte Contemporáneo de Castilla y León), dont les collections se concentrent surtout sur des œuvres des dix dernières années (fig. 1-2).

Dans la même ligne, de nouvelles galeries ont été créées à l'initiative de jeunes spécialistes et, événement plus important encore, des quartiers et même des villes se sont reconstruits autour de nouveaux projets de musées d'art contemporain. Nombre de ces initiatives publiques ont entraîné une réactivation du secteur de l'art et même, dans certains cas, une réhabilitation urbaine. Parallèlement à l'apparition de musées et de salles d'exposition, les collections d'art contemporain ont été consolidées, notamment grâce aux propositions affluantdesmunicipalités, descommunautésautonomesetdesconseils généraux, même s'il faut bien reconnaître qu'une véritable politique d'acquisition leur fait souvent défaut. On pourrait inscrire dans le même élan pour une meilleure connaissance et une plus grande visibilité de l'art contemporain espagnol hors de ses frontières, et pour un échange accru avec les artistes et les professionnels étrangers, la prolifération de biennales dans différentes régions espagnoles et la politique efficace de la Sociedad Estatal de Acción Exterior (SEACEX), qui rend cet échange plus durable qu'une simple "exportation » d'expositions. 
Les efforts des institutions publiques aussi bien que des fondations se concentrent actuellement sur la création de solides réseaux de formation, qui constituent certainement l'une des grandes carences de ce pays dans le domaine de l'art contemporain, pour lequel on doit espérer des politiques à long terme. II serait incontestablement nécessaire de créer des bourses destinées aux jeunes artistes et chercheurs au sein du pays mais aussi et surtout à l'étranger. Certes, ces dix dernières années, de nombreuses initiatives visant à la formation d'artistes et, plus récemment, de commissaires, d'administrateurs ou même de chercheurs, ont été encouragées, comme celles, remarquables, de deux institutions privées : la fondation Marcelino Botín et la fondation Caja Madrid. Mais il reste encore beaucoup à faire : il n'existe toujours pas d'école de conservateurs ou de formation spécialisée pour les commissaires d'expositions. C'est en effet une cause vraisemblable d'un certain déséquilibre entre la pratique et la théorie qu'il faudrait corriger. Maintenant que l'infrastructure est organisée, il est nécessaire de mettre en place des formations solides, de créer des revues scientifiques au sein même des musées, de développer les relations entre ceux-ci et le monde académique et par conséquent les espaces consacrés au débat intellectuel, un terreau susceptible de nourrir les jeunes générations dans une connaissance plus scientifique de l'art actuel [Estrella de Diego].

Estrella de Diego. Ces vingt dernières années, et à la suite des changements politiques survenus dans le pays, le panorama artistique en Espagne a radicalement changé. Comment décririez-vous la situation actuelle et qu'en pensez-vous? Comment la situez-vous dans le contexte international?

Vicente Todolí. Nous avons aujourd'hui plus de musées d'art contemporain que nous n'en avons jamais eu, même si peu ont un poids international et que leurs structures font état d'une dépendance excessive vis-à-vis du pouvoir politique. Ils manquent par conséquent de professionnalisme et sont soumis à des hauts et des bas excessifs. Généralement, leur attention est davantage focalisée sur les contenants que sur les contenus, leur autonomie est insuffisante, leurs instances dirigeantes ne sont pas indépendantes et les
1. Vue de l'exposition Elmgreen \& Dragset, Trying to Remember What We Once Tried to Forget, MUSAC, León, 2009.

2. Vue extérieure du MUSAC, León.
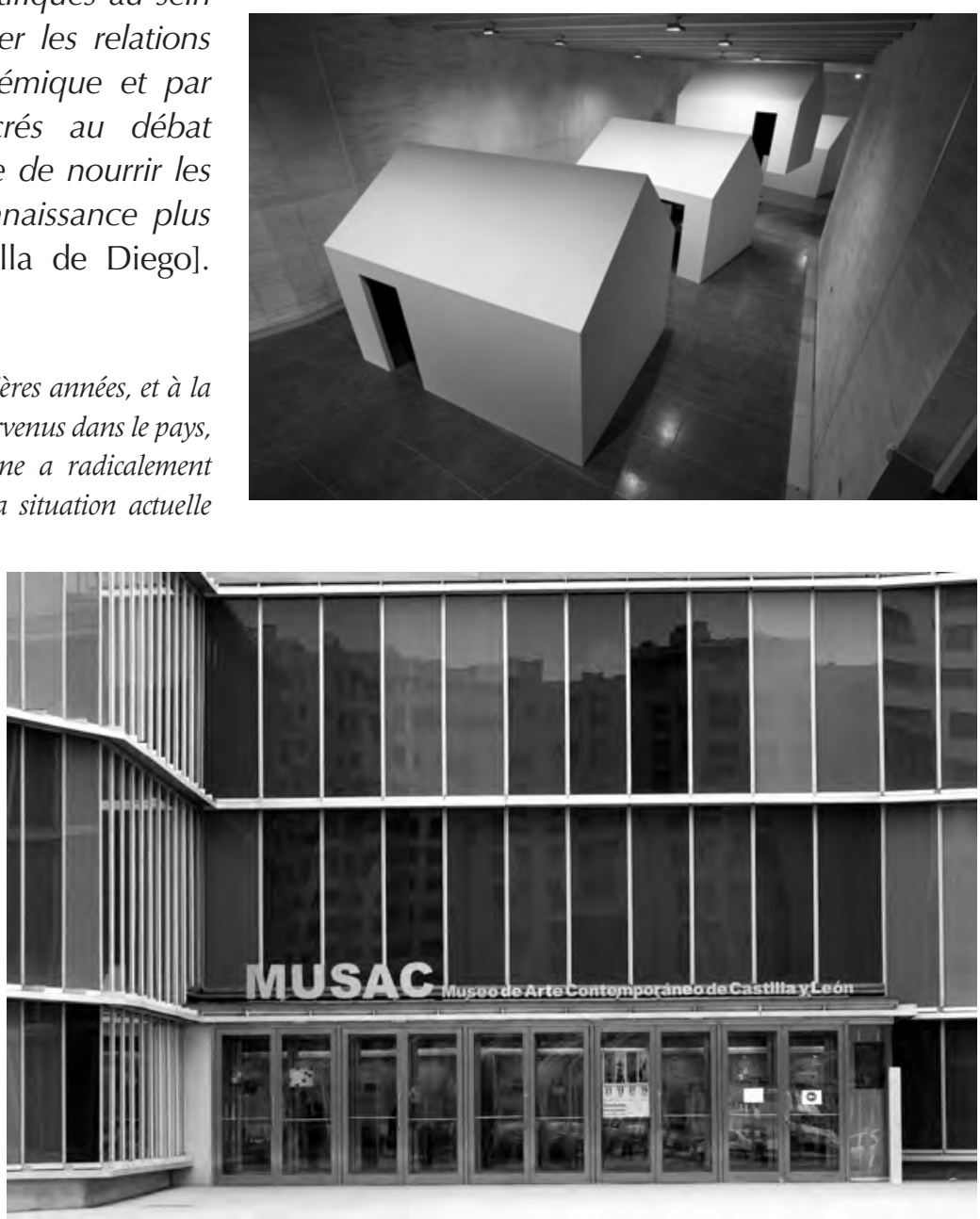
3. L'arrivée du Guernica de Picasso à Madrid en 1981 : réactions de la presse [Rafael FernándezQuintanilla, La odisea del "Guernica " de Picasso, Barcelone, 1981, p. 182-183].

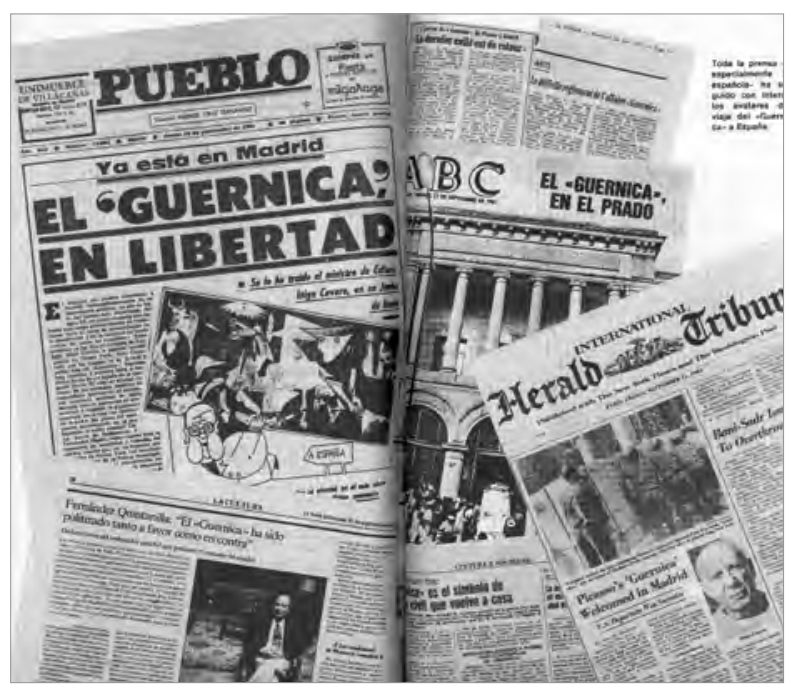

nominations aux postes clés ne sont pas aussi transparentes qu'elles le devraient. En somme, les infrastructures existent, mais globalement elles n'ont pas atteint le niveau qu'on pourrait souhaiter.

Bartomeu Marí. Ce qui a changé radicalement, c'est le nombre d'institutions et de manifestations artistiques : il y a plus de musées et de centres d'art, plus d'expositions, plus de biennales. En revanche, il n'y a pas plus d'écoles, ni de troisième cycle universitaire en art. D'un point de vue qualitatif, on n'observe pas de changement substantiel, mis à part la présence de certains artistes qui, par un acharnement individuel, se distinguent dans un paysage plutôt modeste. C'était toutefois déjà le cas il y a vingt ans. À ce titre, je regrette qu'il n'y ait pas de collections solides et exceptionnelles, même si je reconnais les efforts déployés pour en constituer afin qu'on puisse les apprécier dans... vingt ans peut-être ? L'état des lieux n'est pas désespérant, mais à l'évidence peu enthousiasmant. Quant au panorama international, on peut espérer, étant donné que beaucoup de choses restent à faire, que l'Espagne saura éviter le calme immuable qui domine la majorité des institutions européennes. Alors, on pourra trouver l'avenir enthousiasmant... Pour le moment, le présent est très tranquille et la mer bien plate.

José Guirao. Je pense que le processus a commencé voilà une dizaine d'années, avec la mise en place de la démocratie en Espagne. Le symbole en est I'arrivée du Guernica de Picasso à Madrid en 1981, le « dernier exilé », comme il fut dit dans la presse (1937, Madrid, Museo Nacional Centro de Arte Reina Sofía ; fig. 3). L'accès des socialistes au pouvoir l'année suivante fut également déterminant. Toute une génération voulait combler le retard, rattraper le temps perdu, s'ouvrir au monde et prendre part à la scène internationale. Comme c'est souvent le cas en Espagne, des efforts titanesques furent déployés pour changer la situation après quarante ans de franquisme, mais sans fondement solide, de sorte que la politique, pour le meilleur ou pour le pire, détermina presque tous les changements, en fragilisant de manière préoccupante un panorama dont on se demande toujours s'il ne va pas subitement faire marche arrière. L'IVAM de Valence en est un bon exemple : né dans les années 1980 à l'initiative de spécialistes comme Tomás Llorens, Carmen Alborch et Vicente Todolí, il a été soutenu par une politique et des moyens financiers suffisants pour constituer une magnifique collection d'art, incluant pour la première fois la photographie dans un musée espagnol, et a eu une passionnante programmation d'expositions temporaires. Mais aujourd'hui, vingt ans plus tard, il n'est plus qu'un poussiéreux musée provincial. Le cas du musée Reina Sofía reflète d'une autre manière les particularités d'un système espagnol, où chaque directeur semble avoir un projet différent : en vingt ans d'existence, six directeurs s'y sont succédés, accumulant des changements beaucoup trop nombreux pour un musée en si peu de temps.

Une autre caractéristique espagnole est le lancement de grands projets à l'initiative publique, financés par des administrations nationales, régionales ou locales. A contrario, les projets issus de la sphère privée sont rarissimes, bien que très intéressants pour certains. Le secteur de l'art est ainsi maintenu dans une dépendance étroite envers le monde politique. Enfin, il y a eu une avancée spectaculaire dans la création de nouveaux musées et 
de centres d'art contemporain, comparable à ce qui s'est passé en Allemagne dans les années 1970 et 1980, mais le marché artistique espagnol n'est pas encore consolidé et la présence de nos artistes sur la scène internationale reste fragile. Les galeries d'art dont la programmation est du niveau de celle des autres pays sont à peine une douzaine, et le nombre d'artistes nés de la démocratie présents dans les galeries et les musées étrangers est également très faible.

Estrella de Diego. Un débat omniprésent est celui du soutien officiel à l'art contemporain. En quoi les musées, par leurs différents modes d'acquisition, ont-ils adapté leurs collections à la production de l'art actuel et, globalement, cette création se trouve-t-elle aujourd'hui au niveau des autres pays sur la scène internationale? Pensez-vous que ce pari de l'État pour l'art vivant ait entraîné une certaine négligence envers des époques antérieures, peu étudiées en Espagne? En d'autres termes, et pour utiliser une expression à la mode dans 4. Vue extérieure du MACBA, les années 1980 : avons-nous été "postmodernes " avant même d'avoir été "modernes"?

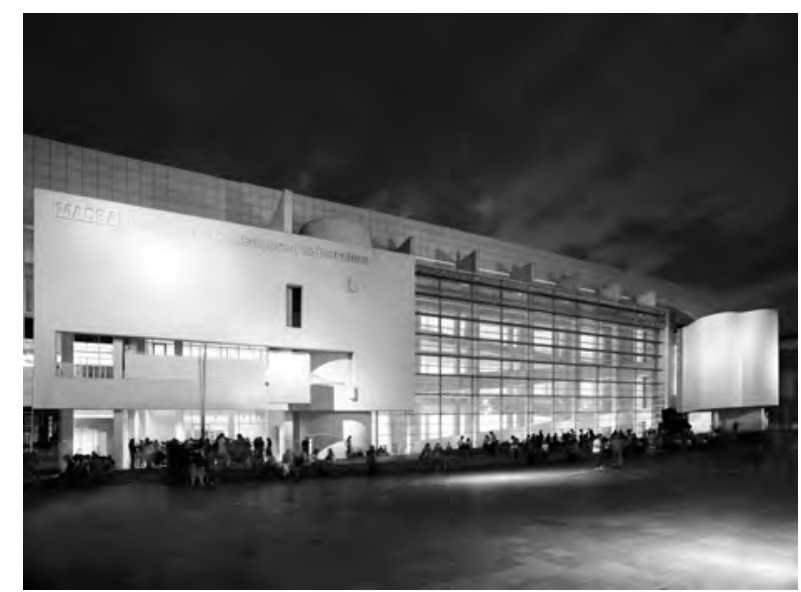
Barcelone.

José Guirao. II faut distinguer les collections permanentes d'art actuel des expositions temporaires. Pour les premières, en réalité seul le MUSAC a eu une politique claire à ce sujet, puisqu'il se définit lui-même comme un musée du présent et que sa collection débute avec des œuvres des années 1990. Même des musées comme le MACBA (fig. 4) n'ont pas suivi une politique d'acquisition dans laquelle l'art actuel primerait sur d'autres périodes comme les années 1950 et 1960. Je pense que le pari en faveur de l'art vivant se reflète davantage dans la programmation d'expositions temporaires, où l'on a eu l'occasion, notamment ces dix dernières années, de voir quasiment tout ce qui se faisait de nouveau dans le monde, presque autant que dans d'autres pays européens ou aux États-Unis. Je me souviens encore, quand j'étais directeur du musée Reina Sofía il y a une dizaine d'années, d'avoir vu le conseil d'administration lui-même refuser I'acquisition d'une œuvre d'Annette Messager alors qu'il avait donné son accord pour une exposition de cette artiste - dont le musée ne possède aujourd'hui encore aucune œuvre. De nombreux musées publics ont acheté des œuvres contemporaines d'artistes espagnols plus par pression du marché local de l'art, qui a toujours beaucoup dépendu des institutions publiques, que dans le cadre d'une politique planifiée pour encourager ce type d'acquisition.

On peut dire en effet que nous avons tenté - parce que je ne crois pas non plus que nous l'ayons réellement été - d'être postmodernes avant même d'être modernes, car il aurait fallu rattraper trop de temps perdu. Toutefois, au début de la mise en place du régime démocratique, a été lancé un plus grand pari encore concernant l'acquisition et l'exposition de l'art moderne et des premières avant-gardes. L'un des fondements de l'IVAM fut de collectionner des œuvres de 1910-1920 qui s'inspiraient de tous les mouvements nés en même temps que le cubisme, ou juste après, ainsi que l'art des années 1930. Dans les années 1980, le Centro Nacional de Exposiciones del Ministerio de Cultura chercha également à faire connaître au public les avant-gardes qui n'avaient pas été vues en Espagne en leur temps. Le musée Reina Sofía, à sa manière, a aussi fait un grand effort pour récupérer les œuvres et les artistes modernes qui avaient un rapport avec l'histoire artistique et culturelle espagnole, avec notamment l'acquisition d'œuvres historiques de Picasso, Miró, Juan Gris, Picabia, Delaunay, etc. Cet effort fut malheureusement laborieux et est resté inachevé. Je pense en définitive que tout s'est passé trop 


\section{L'ESPAGNE}

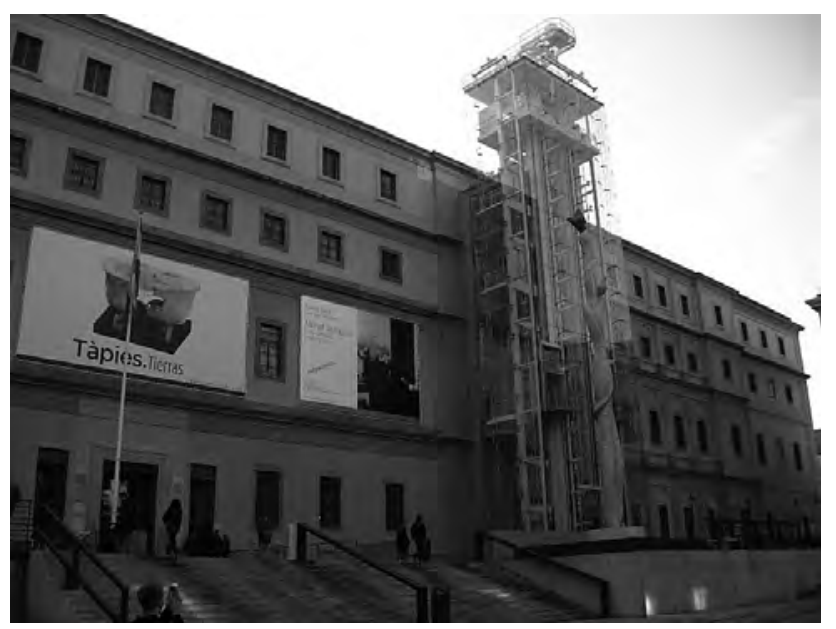

rapidement, que nous n'avons pas eu le temps de digérer notre propre changement historique et que la situation est devenue plus confuse encore avec l'arrivée du XXI ${ }^{e}$ siècle et d'une génération de critiques et d'administrateurs formés sur des bases déjà très éloignées des valeurs et de la connaissance de la modernité.

Bartomeu Marí. Effectivement, les musées ont maintenu un vivier d'artistes qui a augmenté ces vingt dernières années. Mais n'oublions pas que c'est en vingt ans à peine que tout a été créé. Le nouveau réseau de musées a peut-être stimulé la création contemporaine mais il l'a aussi nivelée, unifiée. Par rapport à d'autres pays, l'Espagne manque de formation spécialisée et d'une critique professionnelle. Les artistes espagnols se

5. Vue extérieure du Museo Reina Sofía, Madrid. retrouvent bien moins préparés que la plupart de leurs collègues européens et partent donc désavantagés. Quant à la critique, tant qu'elle ne sera pas professionnalisée, tant qu'on ne pourra pas vivre décemment de l'écriture et de sa diffusion, elle restera prisonnière de sa dépendance envers les institutions qu'elle doit critiquer (musées, galeries, gouvernements autonomes...). On ne peut critiquer objectivement et librement ce qui tôt ou tard nous nourrit.

Les collections d'art moderne n'existent pratiquement pas en Espagne. Celle du musée Reina Sofía, commencée il y a un peu plus de vingt ans, est la seule (fig. 5). De toute manière, la faculté d'inventer de nouvelles visions de la modernité et de les mettre en œuvre à travers nos collections n'est pas le fort de nos musées. II n'existe pas de politique clairement définie dans cette optique et l'État tend, en outre, à centraliser sa politique patrimoniale à Madrid (Prado, Thyssen, Reina Sofía...). On trouve également peu de projets intellectuels liés à des représentations de la modernité. L'approche la plus facile était de commencer avec ce qu'on avait à portée de main, le présent national, et de le justifier en fonction de ce qui se faisait à l'extérieur, en Europe et aux États-Unis.

Vicente Todolí. II est évident que les artistes, pendant leur formation, se nourrissent de ce qu'ils voient. Au cours de leurs études, ils devraient sortir de leur milieu, voyager, voir bien plus que ce que leur environnement géographique leur offre et même vivre un certain temps dans un autre pays, une autre culture. En ce sens, les artistes espagnols bougent peut-être moins.

C'est un problème général : il y a de plus en plus de conservateurs spécialisés en art contemporain et qui s'intéressent peu à l'art antérieur à 1960 (et le connaissent peu, il faut bien le dire). C'est un phénomène récent, qui affecte donc plus les pays dont la majorité des musées ont été créés ces dernières années, mais qui peut durer encore longtemps s'il n'y a pas d'évolution dans la formation des conservateurs.

Estrella de Diego. Quel rôle les artistes, les collectionneurs et les différentes fondations ontils eu dans ce processus?

Bartomeu Marí. Les artistes s'en sont assez bien sortis. Si les collectionneurs sont absents, les fondations le sont tout autant. Seule la fondation Botín offre un soutien à la formation des artistes et à la réalisation de projets personnels. On n'entend plus parler des bourses Fulbright, par exemple, et les aides des gouvernements autonomes sont minimes. 
Vicente Todoli. Peut-être la prolifération de musées et de collections d'art « émergeant » at-elle contribué à un certain déséquilibre au regard de la connaissance et de l'appréciation du passé. On a parfois la sensation que les amateurs d'art d'autrefois ont été remplacés par des «fans » des dernières tendances. Mais, à mon sens, il ne s'agit pas là d'un phénomène exclusivement espagnol.

José Guirao. Les fondations ont joué un rôle immense dans les programmations d'expositions et de débats qui auraient difficilement trouvé leur place au sein des musées et des centres publics. Dans les années 1970, la fondation March à Madrid nous offrit la possibilité de connaître des œuvres essentielles d'artistes d'avant-garde. Je me souviens d'expositions comme celles sur Giacometti, Dubuffet ou Rothko, pour en citer quelques-unes. Plus tard, la fondation Miró de Barcelone a permis de découvrir des aspects de l'œuvre de Miró et ses relations avec les artistes de son entourage. Par la suite, la Fundaciõ Antoni Tàpies à Barcelone ou le Centro José Guerrero à Grenade ont fait un excellent travail en donnant à connaître les dernières créations contemporaines. Quant aux artistes, j'en ai déjà cité certains comme Miró, Tàpies ou Guerrero, dont la générosité a permis aux fondations qui portent leur nom de faire un remarquable travail (fig. 6-7). Parmi les plus jeunes, les noms de Juan Muñoz, Barceló, Cristina Iglesias ou Santiago Sierra, entre autres, ont suscité l'intérêt de musées et de collections internationales pour l'art espagnol, bien que je doute que ceci ait contribué à faire connaître ou à mettre en valeur l'œuvre d'autres artistes espagnols sur la scène internationale. Concernant les collections privées, à ce jour, bien peu sont animées par un projet cohérent. Il reste à voir si leur rôle sera significatif dans la construction d'un panorama général de l'art espagnol ou si elles resteront au stade de simples tentatives, incapables de transcender le goût de leurs propriétaires.

Estrella de Diego. Les musées ont parcouru une longue route pour en arriver où ils en sont

6. Site Internet de la Fundació Tàpies, Barcelone (www. fundaciotapies.com).

7. Site Internet du Centro José Guerrero, Grenade (www. centroguerrero.org). aujourd'hui. L'histoire de l'art espagnole du XX' siècle, à travers la critique et les textes d'opinion, semble avoir, en revanche, encore du chemin à faire pour se "moderniser" et parvenir au niveau des pays qui nous entourent. Peuton parler, selon vous, d'un certain décalage entre théorie et pratique dans la gestion artistique de l'histoire de l'art du XX' siècle?

José Guirao. Sans l'ombre d'un doute, il existe un décalage entre théorie et pratique, alors que ces domaines devraient croître à l'unisson pour assurer un développement équilibré du monde artistique espagnol. Tout d'abord, le monde de la théorie en Espagne se limite au monde universitaire, qui souffre de deux carences fondamentales. En premier lieu, on compte un nombre restreint de chercheurs et d'enseignants qui ont choisi l'art du XXe siècle comme domaine d'études. En second lieu, l'endogamie et le manque de flexibilité du système universitaire espagnol rendent pratiquement impossible d'intégrer des chercheurs et des enseignants, même temporairement, s'ils n'ont pas effectué tout leur parcours au sein de l'université. Enfin, la gestion du champ artistique a elle aussi dû être improvisée, sans formation spécifique préalable, étant donné la rapidité avec laquelle se sont ouverts en nombre nos musées et centres d'art. Le monde académique a besoin de plus de flexibilité structurelle et de moyens économiques, et le secteur administratif d'un plus grand bagage théorique.

Bartomeu Marí. II n'y a pas de pratique historiographique sur le XXe siècle en Espagne, à moins qu'elle ne débute tout juste et qu'on n'en voit pas encore les fruits. Mais en général, la pensée, que ce soit la connaissance ou l'analyse de la
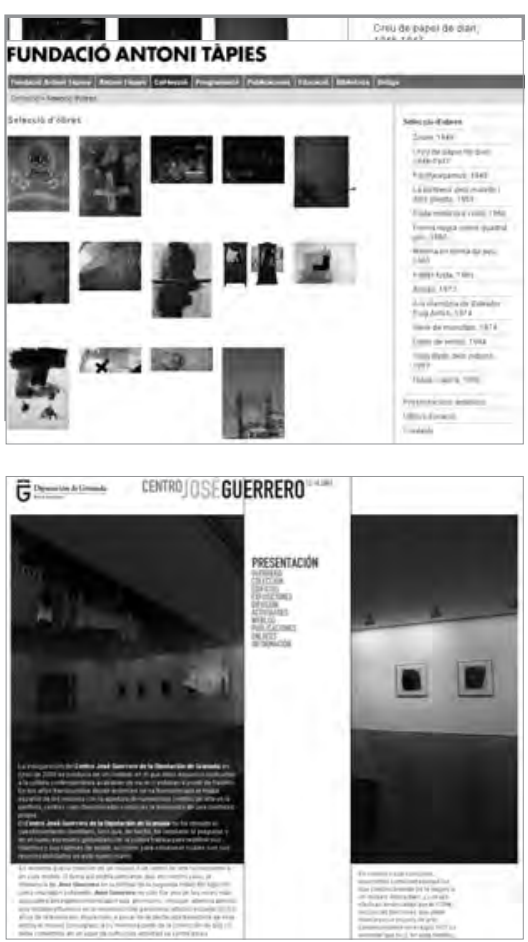
création artistique actuelle, est inexistante. On ne la voit nulle part, elle ne fait pas partie de I'opinion publique et ne jouit d'aucun prestige. II n'y a pas de bourses spécifiques pour les étudiants dans ce domaine et le nombre de thèses doctorales consacrées à l'art actuel (ou à l'art du XXe siècle) est ridiculement bas.

Vicente Todolí. Au Royaume-Uni, il y a un certain équilibre entre le monde académique et/ou critique et le monde muséal, qui collaborent sur de nombreux projets. D'ailleurs, la Tate a récemment inauguré un département de recherche dont le statut est similaire à celui de n'importe quelle université. En Espagne, effectivement, un échange plus fluide dans un sens comme dans l'autre, entre le monde académique et le monde critique serait souhaitable, pour le bénéfice de chacun.

Estrella de Diego. Il n'existe aucun directeur de musée issu du monde académique, ou inversement, comme c'est le cas aux États-Unis ou dans d'autres pays européens. Ce manque d'échanges pourrait-il expliquer le développement moindre de l'histoire de l'art d'un point de vue théorique? De quelle façon une meilleure communication entre les deux mondes pourrait-elle être mise en place et quels en seraient les avantages pour chacun?

José Guirao. Les mécanismes et procédures de gestion sont très différents de ceux de la recherche, et d'ailleurs les directeurs de musées venant du monde académique ont eu du mal à les comprendre. J'imagine que, dans le cas inverse, des difficultés se présenteraient aussi. Il faudrait résoudre deux problèmes : $d$ 'une part pouvoir assurer une professionnalisation du monde de la gestion qui ne soit pas seulement pratique, comme c'est le cas actuellement, et, d'autre part, accorder une plus grande perméabilité au monde académique afin qu'il soit capable d'intégrer l'univers de la gestion dans ses développements. Je pense qu'une fois cela fait, un échange fructueux serait possible, dont la carence actuelle appauvrit les deux parties.

Bartomeu Marí. Je ne pense pas que le problème vienne du fait que les directeurs des institutions ne sont pas issus du monde académique. Si vous voulez dire que les directeurs de musées ont un niveau académique peu élevé, c'est en effet souvent le cas, même s'il y a des exceptions. Les directeurs des trois principaux musées madrilènes ont un très haut niveau d'études. D'autres sont des gestionnaires ou des gérants. Certains, comme moi, sont autodidactes. Quoi qu'il en soit, cela ne devrait pas empiéter sur une action de qualité, fondée sur la prise de risque et des idées originales et audacieuses. Le problème, à mon sens, vient du dysfonctionnement des différents acteurs (personnes et institutions artistiques et politiques, médias) qui composent le contexte artistique qui est le nôtre.

Vicente Todolí. Peu importe qu'un directeur de musée ait une expérience dans l'enseignement, ou qu'un professeur d'histoire de l'art ait préalablement exercé comme conservateur ou directeur de musée. La recherche et la diffusion de la culture sont des points fondamentaux dans les deux domaines. Cela dit, il serait fort salutaire de développer une meilleure communication entre les deux, orientée sur des projets spécifiques et pas nécessairement de façon permanente.

Nota bene : ce texte résulte de l'envoi de questions aux participants et d'échanges de courriels. 